

\section{PROPIEDADES ENERGÉTICAS DE TECNOLOGÍAS DE VENTANAS EN ARGENTINA}

ENERGY PROPERTIES OF WINDOW TECHNOLOGIES IN ARGENTINA

\author{
MAUREEN DE GASTINES \\ Doctora \\ Consejo Nacional de Investigaciones Científicas y Técnicas \\ Instituto de Ambiente, Hábitat y Energía (INAHE) \\ Mendoza, Argentina \\ https://orcid.org/0000-0002-0357-9375 \\ mdegastines@mendoza-conicet.gob.ar
}

\author{
ANDREA ELVIRA PATTINI \\ Doctora \\ Consejo Nacional de Investigaciones Científicas y Técnicas \\ Directora del Instituto de Ambiente, Hábitat y Energía (INAH) \\ Mendoza, Argentina \\ https://orcid.org/0000-0001-6305-1268 \\ apattini@mendoza-conicet.gob.ar
}

\section{RESUMEN}

Las ventanas influyen de manera significativa en las demandas energéticas para calefacción, refrigeración e iluminación de los edificios. En este trabajo, se caracterizan las propiedades energéticas de las tecnologías de ventanas disponibles en Argentina, mediante el cálculo de sus índices K, FS y TV (transmitancia térmica, factor solar y transmitancia visible). Se analiza, además, el impacto que tienen diversos parámetros, como las dimensiones de la abertura, la tipología, el material y la absortancia solar del marco, sobre los índices mencionados. Los casos de estudio se seleccionaron cuidadosamente para asegurar una buena representatividad de la muestra analizada, utilizando, en particular, el método de análisis de conglomerados. El cálculo de los índices se realizó con el apoyo

de los programas WINDOW 6.3 y THERM 6.3. Los resultados indican que los valores de K están comprendidos entre 1.88 y $6.00 \mathrm{~W} / \mathrm{m} 2 \mathrm{~K}$, mientras que los valores de FS varían entre 0.08 y 0.80 , y los de TV entre 0.03 y 0.80 , según el material del marco, la tipología y el tipo de vidriado.

\section{Palabras clave}

ventanas, índices, energía, desempeño, simulación.

\section{ABSTRACT}

Windows significantly impact the energy demand for heating, cooling and lighting in buildings. This study characterizes the energy properties of window technologies available in Argentina by calculating their thermal transmittance (U), solar heat gain coefficient (SHGC) and visible transmittance

(VT). Additionally, the impact of different parameters like window opening dimensions, typology,

frame material and solar absorptance on the mentioned indices is analyzed. Case studies were selected carefully using cluster analysis methods in order to ensure that the sample examined was representative. The calculation of the U, SHGC and VT indices was carried out using WINDOW 6.3 and THERM 6.3 software. The results indicate that $U$-values are between 1.88 and $6.00 \mathrm{~W} / \mathrm{m} 2 \mathrm{~K}$, while SHGC values vary between 0.08 and 0.80 , and VT values are between 0.03 and 0.80 , depending on frame material, typology and glazing characteristics. 


\section{INTRODUCCIÓN}

Las ventanas son protagonistas en los intercambios térmicos y lumínicos entre el exterior y los espacios interiores de los edificios, y por ello impactan de manera significativa en las demandas energéticas para calefacción, refrigeración e iluminación de los mismos (Alam y Islam, 2016; Sharda y Kumar, 2016; Thalfeldt, Kurnitski y Voll, 2016; Alwetaishi, 2017; Trzaski y Rucinska, 2015). Los índices que caracterizan estos flujos energéticos a través de las ventanas son la transmitancia térmica ( $K$, también comúnmente designada por la letra U), el factor solar (FS) y la transmitancia visible (TV). El factor K expresa el flujo de calor entre el lado más cálido y el otro lado (más frío) de la carpintería, por unidad de área y grado de diferencia de temperatura. El factor solar es la fracción de la irradiancia solar incidente sobre una ventana que ingresa al espacio interior. La transmitancia visible es una propiedad óptica que indica la cantidad de luz visible transmitida a través del vidriado.

Si bien existen numerosos estudios sobre las ventanas de alta performance (Granzotto et al., 2017; Jelle et al., 2012; Buratti, Moretti y Zinzi, 2017) y las estrategias para mejorar la transmitancia térmica de los marcos (Appelfeld, Hansen y Svendsen, 2010; Lechowska, Schnotale y Baldinelli, 2017; Van Den Bossche, Buffel y Janssens., 2015; Zajas y Heiselberg, 2014; N. Cardinale, Rospi y T. Cardinale, 2015), que suelen ser el principal puente térmico de las carpinterías, se encuentra poca información sobre las ventanas de baja eficiencia energética, a pesar de su uso extensivo en varias regiones del mundo, principalmente, en las economías emergentes (International Energy Agency, 2013).

Entre estos últimos destaca un amplio estudio sobre la eficiencia energética de las ventanas utilizadas en India, conducido por Rawal et al. (2013). En él se calcularon las propiedades térmicas y ópticas de un conjunto de ventanas de diferentes características para unas dimensiones estándares, para concluir que el material y la tipología de los marcos tienen un impacto significativo en la performance de las ventanas, resaltando la importancia de evaluar la ventana en su conjunto, y no solamente el vidriado.

Medina Agreda (2009), por su parte, abordó la transferencia de calor en marcos de ventanas diseñados en México. Seleccionó ocho tipos de marcos de ventanas característicos de las edificaciones mexicanas (de aluminio, chapa plegada, madera y PVC) y los analizó mediante el uso del programa de conducción de calor THERM, con un vidrio claro de $6 \mathrm{~mm}$ insertado en ellos (a excepción de los marcos de PVC). Obtuvo así valores de factor $\mathrm{K}$ comprendidos entre $2.5 \mathrm{~W} /$ $\mathrm{m}^{2} \mathrm{~K}$ (abatible de PVC) y $5.0 \mathrm{~W} / \mathrm{m}^{2} \mathrm{~K}$ (corrediza de chapa plegada) para las condiciones climáticas de los diferentes Estados de México. Los valores de factor solar para las ventanas estudiadas se ubicaron entre 0.60 (marco de madera) y 0.69 (corrediza de aluminio).

De Gastines, Villalba y Pattini (2016) caracterizaron las ventanas corredizas de aluminio sin ruptura de puente térmico (RPT) en Argentina y advirtieron que sus valores de transmitancia térmica de marco son muy elevados (9.8 W/ $\mathrm{m}^{2} \mathrm{~K}$ en promedio), debido a su baja compacidad. Combinados con vidrios simples, resultan en carpinterías muy ineficientes, con factores $\mathrm{K}$ del orden de los $7 \mathrm{~W} / \mathrm{m}^{2} \mathrm{~K}$.

En los países como Argentina, donde las ventanas son de baja performance, hay un elevado potencial de ahorro energético asociado a la selección de aberturas. Sin embargo, muchas veces, las decisiones al momento de elegir los componentes de las viviendas se ven influenciadas por cuestiones económicas. Los consumidores se encuentran con opciones de vidriados eficientes y de marcos aislantes, los que pueden encarecer significativamente los componentes. Por lo tanto, surge la duda de conocer el impacto de estas decisiones sobre la eficiencia de la ventana.

En respuesta a esta problemática, se han desarrollado, en algunos países, sistemas de certificación energética de ventanas, que evalúan la eficiencia de las ventanas mediante un balance energético, basado en los índices $\mathrm{K}$ y FS, generalmente calculados para unas dimensiones estándares. Sin embargo, dichos índices varían en función de distintos parámetros, como las dimensiones de la ventana y el ancho del marco, que definen la relación de superficie entre el vidriado y las diferentes secciones del marco.

Otro parámetro que influye en el valor del factor solar de las ventanas es la absortancia solar del marco, que se modifica según el acabado del mismo. Aunque se suela despreciar la contribución del marco al factor solar de la ventana, los marcos de alta conductancia pueden tener un factor solar relativamente elevado, el cual depende además de su absortancia solar (Wright y McGowan, 1999).

El objetivo de este trabajo consiste en establecer un panorama de las propiedades energéticas de las ventanas en Argentina, y analizar el impacto de diferentes parámetros (dimensiones, tipología, material y absortancia solar del marco) sobre los índices térmicos de las ventanas, con el fin de definir criterios para la selección de ventanas energéticamente eficientes.

\section{MÉTODOS}

\section{METOdOLOGÍA DE CÁLCULO DE LOS ÍNDICES ENERGÉTICOS}

La transmitancia térmica de la ventana depende de la transmitancia térmica del marco $\left(K_{m}\right)$, la del vidriado $\left(K_{v}\right)$ y de los efectos de borde $\left(K_{b}\right)$ entre ambas partes. Asimismo, el factor solar y la transmitancia visible se calculan como el promedio ponderado de los índices respectivos del marco y del vidriado.

Para el cálculo del factor $\mathrm{K}$ de los marcos y del borde, se utilizó el programa de cálculo bidimencional de 
transferencias de calor THERM 6.3, una herramienta útil y confiable para el cálculo de la transmitancia térmica (Held, Alías y Jacobo, 2017; De Gastines, Correa y Pattini, 2018) basada en el método de los elementos finitos. Se consideraron cavidades parcialmente ventiladas y un modelo radiativo detallado de ambos lados del marco, de acuerdo a los criterios de simulación descritos en De Gastines, Correa y Pattini (2018). Se calculó el factor solar de los marcos mediante la ecuación siguiente (Wright y McGowan, 1999; De Gastines y Pattini, 2017):

$$
F S_{m}=\alpha_{m} \cdot K_{m} \cdot \frac{X_{o}}{h_{o}}
$$

Donde: $\alpha_{m}$ es la absortancia solar del marco, $X_{\circ}$ es la compacidad exterior del marco (i.e. la fracción entre el ancho del marco y la longitud del perfil) y $h_{0}$ es el coeficiente pelicular exterior.

La transmitancia visible de los marcos es nula. La transmitancia térmica, el factor solar y la transmitancia visible de los vidriados se determinaron con el programa WINDOW 6.3, que permite calcular los índices energéticos de las ventanas mediante algoritmos unidimensionales de transferencia de calor.

\section{Condiciones de borde}

Se definieron condiciones de borde para el cálculo de los índices energéticos. Las mismas deben ser representativas de las condiciones climáticas a las cuales la ventana estará sometida, para que los índices reflejen adecuadamente la magnitud de los flujos de energía a través de la ventana.

De acuerdo al Instituto Geográfico Nacional de Argentina, la población total de Buenos Aires (18,515,235 habitantes) representaba el $46 \%$ de la población total Argentina en el censo de 2010 (40,117,096 habitantes) ${ }^{1}$. Dado que la cantidad de habitantes se relaciona con la cantidad de viviendas $y$, por ende, de aberturas, se definieron las condiciones de borde utilizando los datos climáticos detallados en la norma IRAM 11603 (2012) correspondientes a la localidad de Buenos Aires. Se consideró una temperatura exterior igual a la temperatura media de invierno, una temperatura interior de $21^{\circ} \mathrm{C}$, y unas temperaturas medias radiantes iguales a la temperatura del aire. En cuanto al coeficiente convectivo interior, se seleccionó el coeficiente indicado en la NFRC para ventanas de aluminio $\left(3.29 \mathrm{~W} / \mathrm{m}^{2} \mathrm{~K}\right)$ y se calculó el coeficiente convectivo exterior a partir de la velocidad de viento promedio de invierno, utilizando el modelo de Kimura para cuarto piso $^{2}$ y promediando el coeficiente

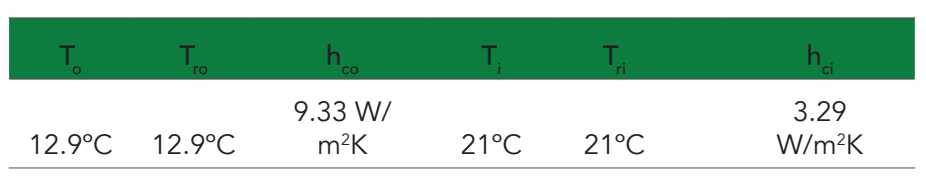

Tabla 1. Condiciones de borde empleadas para el cálculo de los índices energéticos. Fuente: Elaboración de las autoras.

barlovento y sotavento. Dicho modelo fue elegido por ser el que mejor se ajusta a los datos experimentales de Yazdanian y Klems (1994).

\section{SELECCIÓN DE CASOS DE ESTUDIO}

\section{Criterios y metodología de selección}

La selección de la muestra de ventanas se fundamentó en dos criterios principales:

- Relevancia

Se estiman relevantes los productos con protagonismo pasado, actual o futuro en el mercado. Por un lado, se busca incluir en el estudio los productos más vendidos en la actualidad. Por otro, es pertinente tomar en cuenta las tecnologías que fueron predominantes en el pasado, ya que todavía están presentes en gran parte de los edificios existentes. Finalmente, es necesario considerar las tecnologías novedosas, que tienen un potencial de mayor ahorro energético. Estos productos son especialmente relevantes en el marco del estudio propuesto $y$, si bien su uso está limitado todavía, es probable que se difunda durante la próxima década.

- Comportamiento energético

Se busca establecer una muestra representativa del conjunto de combinaciones posibles de índices energéticos K, FS, y TV. Los casos de estudio deben diferenciarse entre ellos y cubrir el rango de eficiencia energética.

De acuerdo a estos criterios, se realizó una selección preliminar de marcos y de vidriados por separado.

\section{Selección preliminar de marcos}

De acuerdo al criterio de relevancia, se seleccionaron varias tipologías de marcos de aluminio sin RPT (ampliamente mayoritario en el mercado actual) pero se incluyeron también alternativas de aluminio con RPT y de PVC, que tienen mayor potencial de ahorro energético. Se contempló, también, una abertura de madera y una abertura de chapa ya que, a pesar de ser tecnologías en vía de desaparición, todavía se encuentran en las construcciones existentes. 


\begin{tabular}{ccccccc} 
Código & Material & Tipología & $\mathrm{H}_{\mathrm{m}}(\mathrm{mm})$ & $\mathrm{X}_{\circ}$ & $\mathrm{X}_{\mathrm{i}}$ & $\mathrm{K}_{\mathrm{m}}\left(\mathrm{W} / \mathrm{m}^{2} \mathrm{~K}\right)$ \\
AC & Aluminio & Corrediza & 99 & 0.560 & 0.604 & 7.37 \\
AA & Aluminio & Abrir & 94 & 0.885 & 0.710 & 5.98 \\
AF & Aluminio & Paño fijo & 45 & 0.818 & 0.625 & 6.99 \\
RC & Alu c/ RPT & Corrediza & 111 & 0.471 & 0.619 & 4.26 \\
RA & Alu c/ RPT & Abrir & 109 & 0.887 & 0.785 & 3.50 \\
PC & PVC & Corrediza & 125 & 0.641 & 0.656 & 2.49 \\
PA & PVC & Abrir & 163 & 0.844 & 0.794 & 2.17 \\
PF & PVC & Paño fijo & 63 & 0.812 & 0.767 & 2.48 \\
CC & Chapa & Corrediza & 67 & 0.348 & 0.362 & 7.18 \\
MA & Madera & Abrir & 103 & 0.757 & 0.715 & 1.88 \\
\hline
\end{tabular}

Tabla 2: Características de los marcos seleccionados, su ancho $\mathrm{H}_{\mathrm{m}^{\prime}}$ sus factores de compacidad $\mathrm{X}_{\mathrm{i}}$ y $\mathrm{X}_{\mathrm{o}^{\prime}}$ y sus índices $\mathrm{K}_{\mathrm{m}}$. Fuente: Elaboración de las autoras.

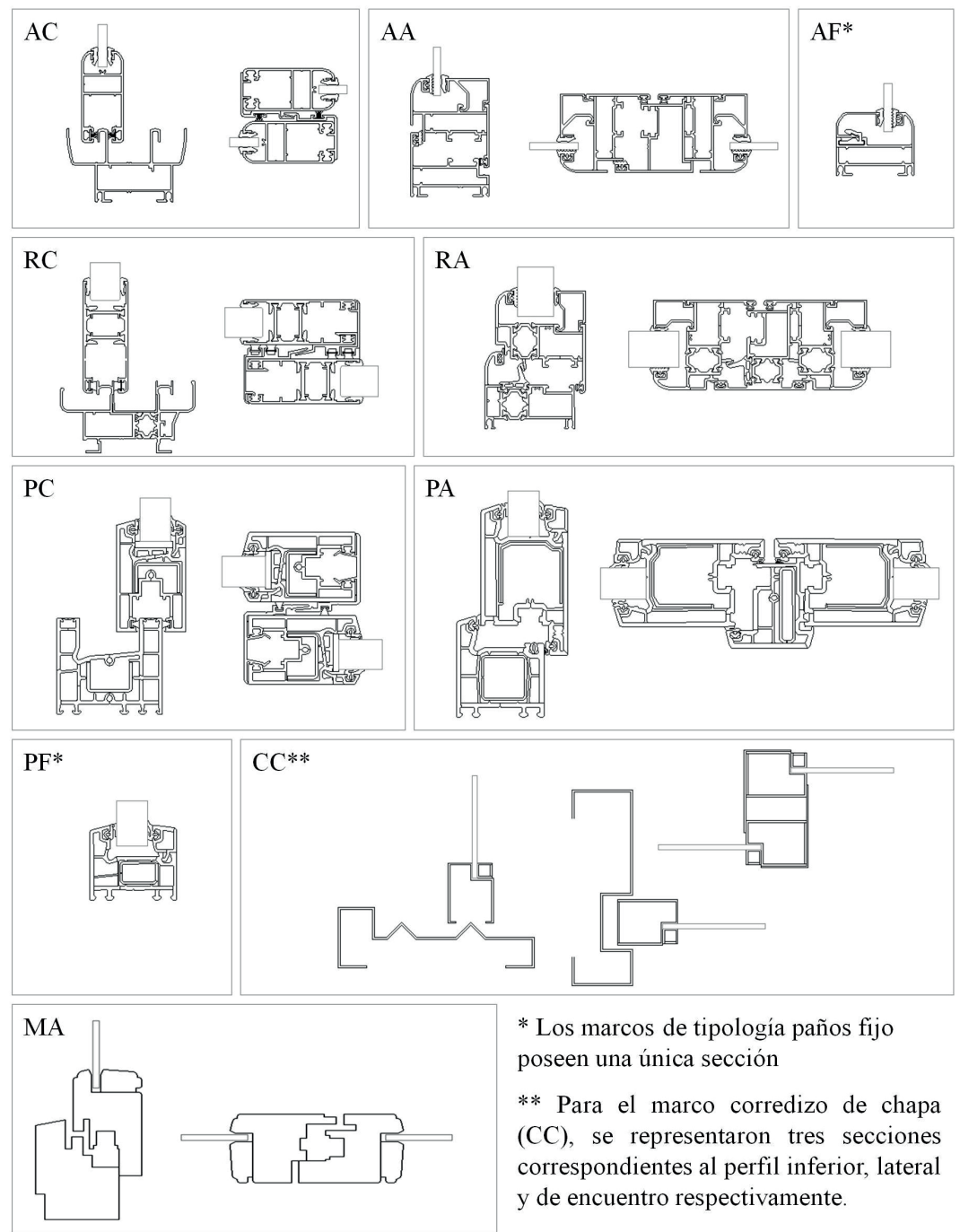

Figura 1. Secciones características (perfil inferior y perfil de encuentro) de los marcos seleccionados. Fuente: Elaboración de las autoras. 


\begin{tabular}{cccccccccccc}
$\mathrm{N}^{0}$ & Elementos & \multicolumn{3}{c}{$\mathrm{K}_{\mathrm{v}}$} & \multicolumn{3}{c}{$\mathrm{FS}_{\mathrm{v}}$} & \multicolumn{3}{c}{$\mathrm{TV}_{\mathrm{v}}$} \\
& & mín. & prom. & máx. & mín. & prom. & máx. & mín. & prom. & máx. \\
1 & 49 & 1.30 & 1.70 & 2.04 & 0.236 & 0.337 & 0.451 & 0.269 & 0.408 & 0.599 \\
2 & 5 & 4.36 & 4.45 & 4.53 & 0.817 & 0.839 & 0.873 & 0.869 & 0.885 & 0.899 \\
3 & 12 & 1.72 & 2.14 & 2.63 & 0.197 & 0.248 & 0.296 & 0.070 & 0.146 & 0.196 \\
4 & 15 & 2.45 & 2.61 & 2.73 & 0.387 & 0.447 & 0.530 & 0.320 & 0.439 & 0.587 \\
5 & 13 & 1.71 & 2.26 & 2.66 & 0.645 & 0.719 & 0.779 & 0.730 & 0.785 & 0.840 \\
6 & 18 & 1.35 & 1.92 & 2.73 & 0.390 & 0.498 & 0.638 & 0.495 & 0.609 & 0.684 \\
7 & 3 & 4.02 & 4.20 & 4.49 & 0.359 & 0.388 & 0.405 & 0.085 & 0.167 & 0.217 \\
8 & 6 & 4.30 & 4.46 & 4.49 & 0.498 & 0.586 & 0.653 & 0.370 & 0.544 & 0.764 \\
\hline
\end{tabular}

Tabla 3. Características de los ocho conglomerados: cantidad de elementos, valores mínimos, promedio y máximo de $\mathrm{K}_{\mathrm{v}^{\prime}} \mathrm{FS}_{\mathrm{v}}$ y $\mathrm{TV}_{\mathrm{v}}$. Fuente: Elaboración de las autoras.

En cuanto a la selección de tipologías, las corrediza, de abrir (rotación sobre eje vertical lateral) y paño fijo (hoja sellada al marco) son las más frecuentes.

Las tipologías guillotina y proyectante (rotación sobre eje horizontal), además de ser menos vendidas, generalmente están asociadas a dimensiones de abertura más pequeñas, por lo que su comportamiento energético cobra menos relevancia a escala edilicia. En consecuencia, no se incluyeron en el análisis.

A partir del criterio energético, se procuró cubrir el rango posible de valores de transmitancia térmica (índice más relevante para los materiales opacos). Esto se logró variando las conductividades térmicas de los materiales y los niveles de compacidad de los perfiles. En particular, se seleccionó un marco de aluminio sin RPT corredizo (material muy conductor y perfil poco compacto) y un marco de PVC de paño fijo (poco conductor y muy compacto), para cubrir todo el rango existente de transmitancia térmica.

Se buscó variar el ancho del perfil, de manera de tener la posibilidad de comparar diferentes proporciones entre superficies opaca y transparente, para unas dimensiones dadas de ventana. En el caso del aluminio y del PVC, se escogió una tipología paño fijo (ancho mínimo) y otra (de abrir o corrediza) de ancho más elevado.

La Figura 1 muestra los perfiles característicos de los marcos seleccionados, mientras que la Tabla 2 indica sus características (ancho $\mathrm{H}_{\mathrm{m}^{\prime}}$ calculado como el promedio ponderado de los anchos de cada sección del marco, y factores de compacidad) y los valores de $K_{m}$ para las dimensiones $1.5 \times 1.2 \mathrm{~m}$ y las condiciones de entorno definidas en la Tabla 1. Se comprueba que los valores de $\mathrm{K}_{\mathrm{m}}$ están bien distribuidos (en el rango [1.88; 7.37]), así como los anchos de marcos (45 a $163 \mathrm{~mm}$ ) y valores de compacidad (entre 0.348 y 0.885 ).

\section{Selección preliminar de vidriados}

Los vidriados más frecuentemente utilizados son el vidriado simple float claro de $4 \mathrm{~mm}$, el laminado $3+3$ (dos paños de $3 \mathrm{~mm}$ separados por una lámina de butiral de polivinilo o PVB) y el doble vidriado hermético (DVH) float claro 4-94 (dos paños de $4 \mathrm{~mm}$ de espesor separados por una cámara de aire de $9 \mathrm{~mm}$ de espesor). No obstante, existe una gran diversidad de vidrios disponibles, que cambian significativamente en cuanto a sus propiedades térmicas y lumínicas. Con el propósito de limitar la cantidad de pares marco/vidriado a simular, se realizó un análisis de conglomerados para diferenciar los vidrios disponibles y seleccionar casos bien distintos entre ellos.

Se emplearon los datos de la empresa VASA ${ }^{3}$ para definir la lista de vidriados disponibles en el mercado en las configuraciones siguientes: 4, 6, 8, 3+3, 4+4, 4-9-4, 4-9$3+3$, 4-12-4, 6-9-6, 6-12-3+3. Se obtuvo un total de 121 vidriados. Luego, se simularon los vidriados en WINDOW, utilizando las condiciones de entorno definidas en la tabla 1 , y se calcularon sus índices $\mathrm{K}_{\mathrm{v}}, \mathrm{FS}_{\mathrm{v}}$ y $\mathrm{TV}_{\mathrm{v}}$.

\section{Análisis de conglomerados.}

Mediante un análisis de conglomerados jerárquico en Infostat, se clasificaron los 121 vidriados en ocho conglomerados, en base a las tres variables estandarizadas $\mathrm{K}_{\mathrm{v},} \mathrm{FS}_{\mathrm{v}}$ y $\mathrm{TV}_{\mathrm{v}}$ y aplicando la distancia euclidiana y el método del encadenamiento promedio. La Tabla 3 resume las características de los ocho conglomerados obtenidos aquí. 


\begin{tabular}{ccccccccc} 
Cód. & Designación & SV/DVH & Tono & Ancho & Congl & Kv & FSv & TVv \\
$\# 1$ & Float 4 & SV & claro & $4 \mathrm{~mm}$ & 2 & 4.53 & 0.873 & 0.899 \\
$\# 2$ & Tints ab 6 & SV & azul & $6 \mathrm{~mm}$ & 8 & 4.49 & 0.559 & 0.526 \\
$\# 3$ & CLSTB120 6 & SV & turquesa & $6 \mathrm{~mm}$ & 7 & 4.08 & 0.399 & 0.217 \\
$\# 4$ & Low-E 6 & SV & claro & $6 \mathrm{~mm}$ & 5 & 2.56 & 0.717 & 0.819 \\
$\# 5$ & Eclipse eg 6 & SV & verde & $6 \mathrm{~mm}$ & 4 & 2.73 & 0.401 & 0.478 \\
$\# 6$ & Float 4-9-4 & DVH & claro & $17 \mathrm{~mm}$ & 5 & 2.66 & 0.778 & 0.813 \\
$\# 7$ & CLKNT164 6-9-6 & DVH & neutro & $21 \mathrm{~mm}$ & 6 & 1.96 & 0.470 & 0.572 \\
$\# 8$ & Tints ab 6-9-6 & DVH & azul & $21 \mathrm{~mm}$ & 4 & 2.63 & 0.430 & 0.467 \\
$\# 9$ & CLST120 Low-E 6-9-6 & DVH & plateado & $21 \mathrm{~mm}$ & 3 & 1.95 & 0.232 & 0.169 \\
$\# 10$ & Tints Low-E ab 6-9-6 & DVH & azul & $21 \mathrm{~mm}$ & 1 & 1.78 & 0.364 & 0.433 \\
\hline
\end{tabular}

Tabla 4. Vidriados seleccionados con sus características (simple vidriado o doble vidriado hermético, tono, ancho, número de conglomerado) e índices $\mathrm{K}_{\mathrm{v}^{\prime}} \mathrm{FS}_{\mathrm{v}}$ y $\mathrm{TV}_{\mathrm{v}}$. Fuente: Elaboración de las autoras.

Los conglomerados 1,4 y 6 son los que más elementos contienen y sus rangos de valores $\mathrm{K}_{v^{\prime}} \mathrm{FS}_{\mathrm{v}}$ y $\mathrm{TV}_{\mathrm{v}}$ se solapan. El conglomerado 2 contiene los vidriados simples float claros y se caracteriza por sus altos valores de $\mathrm{K}_{v}$ (superiores a $4.36 \mathrm{~W} / \mathrm{m}^{2} \mathrm{~K}$ ) y $\mathrm{FS}_{\mathrm{v}}$ (superiores a 0.869). Los conglomerados 7 y 8 están compuestos por vidriados simples reflectivos y de color, de $\mathrm{K}_{v}$ superiores a $4.02 \mathrm{~W} /$ $\mathrm{m}^{2} \mathrm{~K}$, y diferenciados por sus valores de $\mathrm{FS}_{\mathrm{v}}$ (inferiores a 0.405 para el conglomerado 7 , comprendidos entre 0.485 y 0.653 para el 8). El conglomerado 3 incluye los $\mathrm{DVH}$, con un $\mathrm{K}_{v}$ inferior a $2.73 \mathrm{~W} / \mathrm{m}^{2} \mathrm{~K}$ y una $\mathrm{TV}_{v}$ muy baja (inferior a $0.196)$. Y el conglomerado 5 contiene los vidriados con un $\mathrm{K}_{\mathrm{v}}$ inferior a $2.73 \mathrm{~W} / \mathrm{m}^{2} \mathrm{~K}$ y un $\mathrm{FS}_{\mathrm{v}}$ superior a 0.645 .

Se intentó seleccionar como mínimo un vidriado dentro de cada conglomerado para obtener una muestra representativa del conjunto de vidriados disponibles en el mercado. De esa forma, se optó prioritariamente por el simple vidriado float claro de $4 \mathrm{~mm}$ y el DVH float claro 4-9-4, que pertenecen a los conglomerados 2 y 5 , respectivamente, por constituir las opciones más vendidas.

Para el resto de los conglomerados, se elaboraron matrices de distancia euclidiana con el objetivo de elegir el elemento más representativo de cada conglomerado (es decir, el que presenta la menor distancia con el resto de los elementos del conglomerado).

Los conglomerados 4, 5 y 6 incluyen principalmente DVH, pero también algunos vidriados simples. Considerando que existen en el mercado marcos que admiten únicamente vidriados simples, es interesante poder lograr propiedades similares a las de un DVH con un vidriado simple. Por este motivo, se seleccionó el vidrio Low-e de $6 \mathrm{~mm}$ (conglomerado 5) y el vidrio "Eclipse evergreen" de $6 \mathrm{~mm}$ (conglomerado 4), adicionalmente a los DVH más representativos de cada conglomerado. En total, se obtuvo una muestra de diez vidriados, cuyas propiedades se detallan en la Tabla 4.

\section{Metodología de análisis}

A partir de las muestras de marcos y vidriados seleccionadas, se construyeron ventanas teniendo en cuenta la compatibilidad entre el galce ${ }^{4}$ del marco y el ancho del vidriado, y como también los diferentes tamaños de abertura $(0.6 \times 1.1 \mathrm{~m}, 1.5 \times 1.2 \mathrm{~m}$ y $1.8 \times 1.5 \mathrm{~m})$. Asimismo, se consideraron dos valores de absortancia solar de marcos: 0.3 (marcos blancos) y 0.9 (marcos oscuros).

Por último, se calcularon los índices K, FS y TV de las ventanas así definidas, utilizando las condiciones de entorno establecidas anteriormente (Tabla 1).

\section{RESULTADOS}

Las Figuras 2, 3 y 4 ilustran las variaciones de los diferentes índices en función de las dimensiones de la ventana, considerando una absortancia solar de 0.3 (pintura blanca) para todos los marcos. Exponen, además, los valores de los índices correspondientes al marco y al vidriado, respectivamente.

Se observa que los valores de transmitancia térmica del conjunto tecnológico varían entre 1.88 y $6.00 \mathrm{~W} / \mathrm{m}^{2} \mathrm{~K}$. Para 


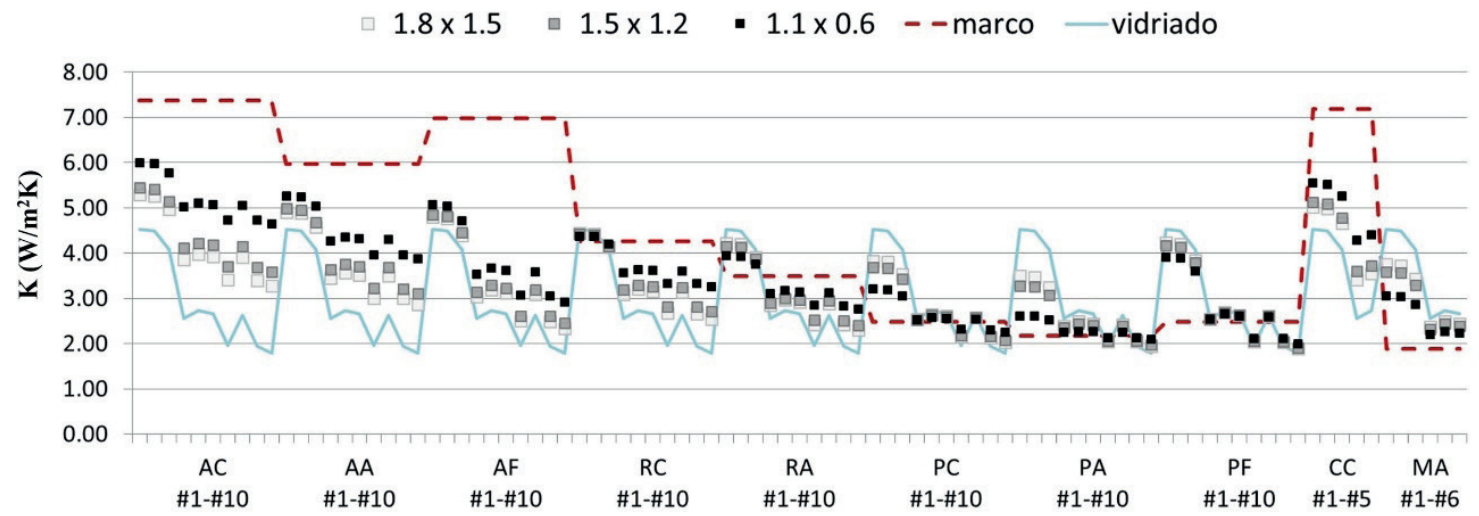

Figura 2. Valores de K del conjunto tecnológico, para diferentes dimensiones de ventana. Fuente: Elaboración de las autoras.

$\square 1.8 \times 1.5 \square 1.5 \times 1.2 \quad \cdot 1.1 \times 0.6--$ marco $\square$ vidriado

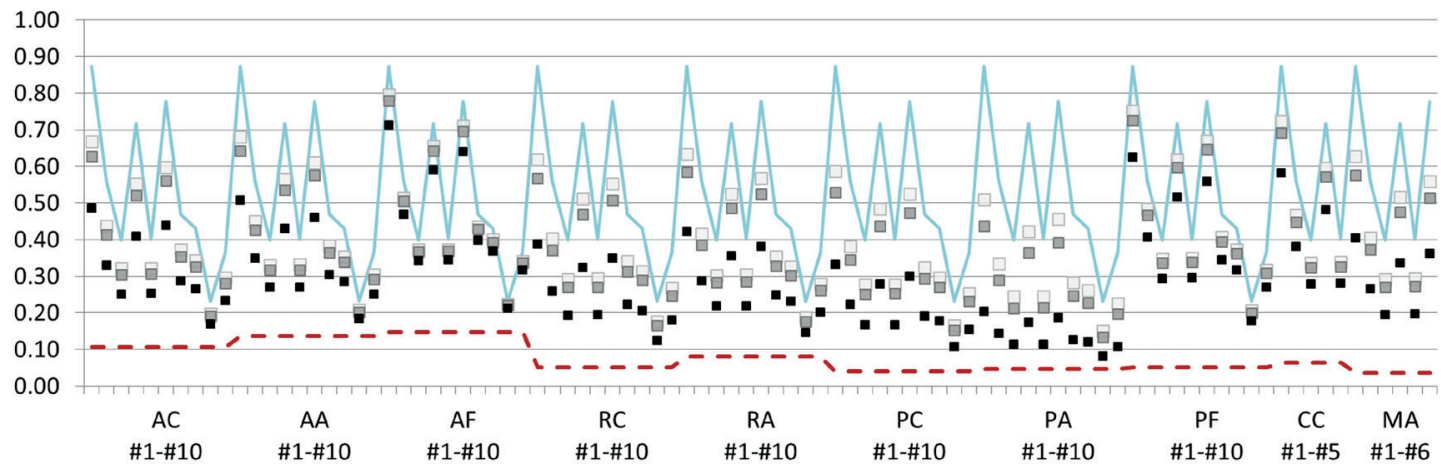

Figura 3. Valores de FS del conjunto tecnológico, para diferentes dimensiones de ventana. Fuente: Elaboración de las autoras.

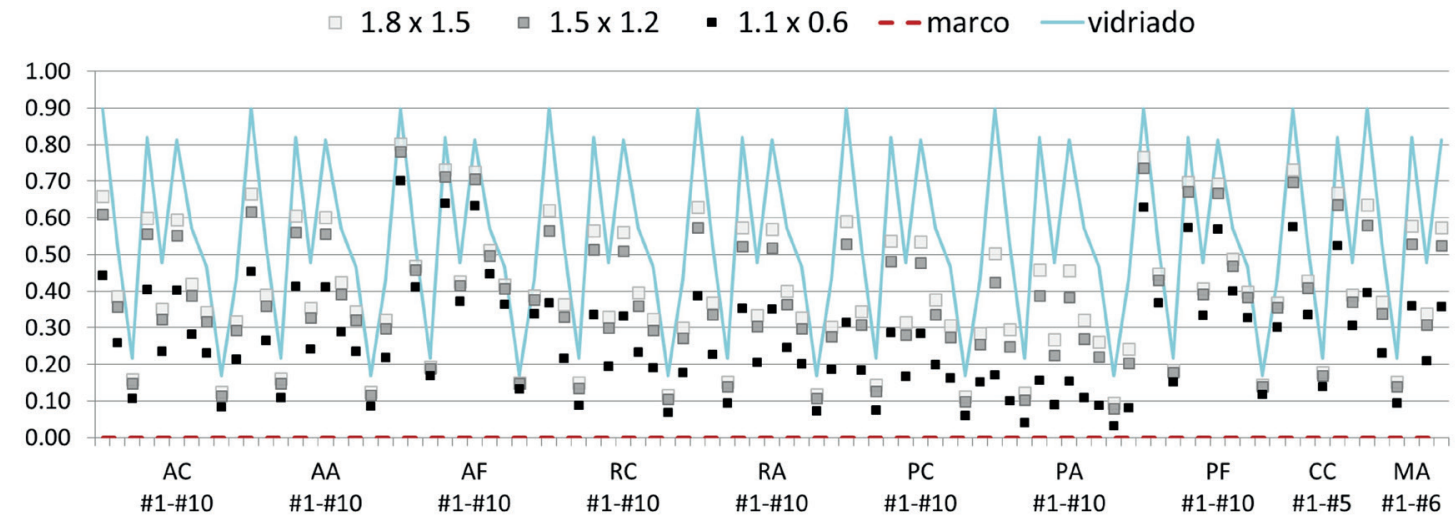

Figura 4. Valores de TV del conjunto tecnológico, para diferentes dimensiones de ventana. Fuente: Elaboración de las autoras. 
$\square \mathrm{abs}=0.3 \quad \circ \mathrm{abs}=0.9$

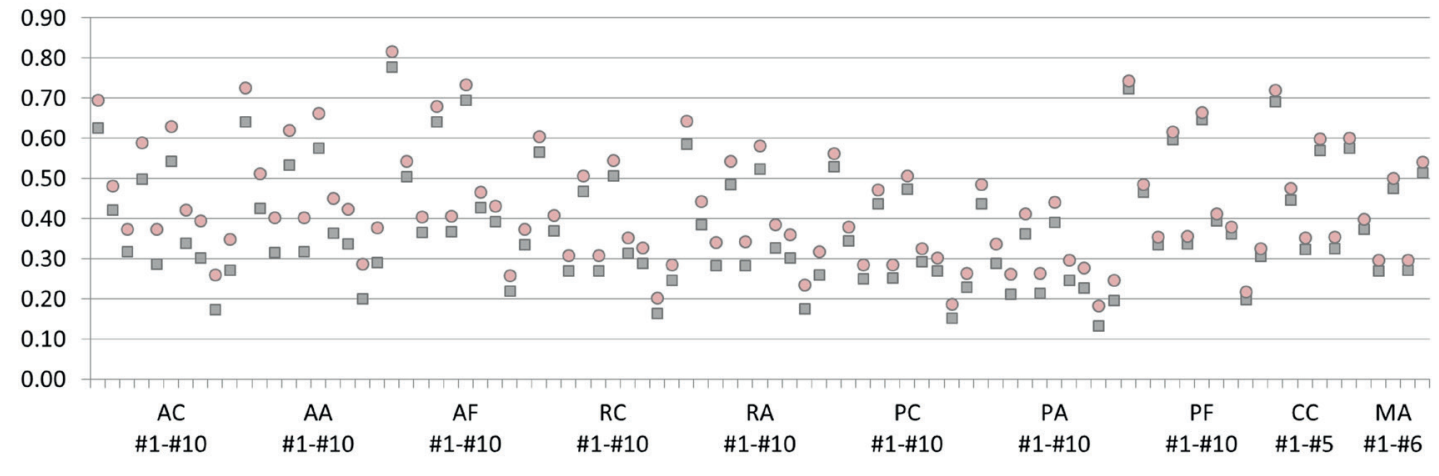

Figura 5. Impacto de la absortancia solar del marco sobre los valores de FS de las ventanas. Fuente: Elaboración de las autoras.

unas determinadas dimensiones de abertura, los valores máximos de $\mathrm{K}$ corresponden a las ventanas con marco de aluminio y chapa asociado a los vidriados \#1, \#2 y \#3 (compuestos por materiales de elevada conductividad). Los valores mínimos se obtienen para las ventanas con marco de PVC combinado con los vidriados \#7, \#9 y \#10 (DVH con capa reflectiva, de control solar y/o baja emisividad).

La tipología influye de manera significativa en el valor de K. En el caso del aluminio, la tipología paño fijo es la que tiene valores de $\mathrm{K}$ más bajos debido a su ancho reducido $\left(K_{v}\right.$ es inferior a $\left.K_{m}\right)$, mientras que la tendencia se invierte para los marcos de PVC asociados a vidrios poco aislantes $\left(K_{v}\right.$ es superior a $\left.K_{m}\right)$.

El valor del factor $\mathrm{K}$ se ve influenciado por las dimensiones de la ventana principalmente en los casos que presentan un gran contraste entre $\mathrm{K}_{\mathrm{v}}$ y $\mathrm{K}_{\mathrm{m}^{\prime}}$ alcanzando diferencias relativas del orden del $30 \%$ entre las dimensiones $0.6 \times$ $1.1 \mathrm{~m}$ y 1.8 x $1.5 \mathrm{~m}$ (ventanas AC7-9-10 y PA1-2-3). Sin embargo, estas diferencias se mantienen por debajo del $8 \%$ entre las dimensiones $1.5 \times 1.2 \mathrm{~m}$ y $1.8 \times 1.5 \mathrm{~m}$.

En promedio, la estrategia que consiste en reemplazar un marco de aluminio por un marco de PVC de misma tipología, significa una reducción del valor de $\mathrm{K}$ del $42 \%$ para ventanas de dimensiones $0.6 \times 1.1 \mathrm{~m}$, y del $26 \%$ para ventanas de $1.8 \times 1.5 \mathrm{~m}$. Igualmente, la estrategia que consiste en reemplazar el simple vidriado claro por un DVH float claro resulta en una mejora en el factor $\mathrm{K}$ del $22 \%$ para las dimensiones $0.6 \times 1.1 \mathrm{~m}$, y del $32 \%$, para ventanas de $1.8 \times 1.5 \mathrm{~m}$; y reemplazando el simple vidriado claro por el doble vidriado de control solar y low-e (\#10), se logra una reducción de $\mathrm{K}$ del $31 \%$ y del $46 \%$ para las dimensiones $0.6 \times 1.1 \mathrm{~m}$ y $1.8 \times 1.5 \mathrm{~m}$, respectivamente. Por lo tanto, si el usuario, para bajar la transmitancia de su ventana (típicamente en clima frío), tuviera que elegir entre un vidriado eficiente y un marco eficiente, por cuestiones económicas, la mejor estrategia depende de las dimensiones de la abertura. En los ejemplos analizados, se observó que en el caso de una ventana chica $(0.6 \times 1.1$ $\mathrm{m}$ ) el material y la tipología del marco determinan en gran medida el valor de la transmitancia térmica de la ventana completa, mientras que en el caso de una ventana grande $(1.8 \times 1.5 \mathrm{~m})$, conviene invertir en un vidriado eficiente.

En cuanto al factor solar y a la transmitancia visible, su valor máximo es de 0.80 (ventana AF1, dimensiones 1.8 × 1.5) y su valor mínimo, de 0.08 y 0.03 , respectivamente (ventana PA9, dimensiones $0.6 \times 1.1 \mathrm{~m}$ ). Para unas dimensiones dadas, los valores más altos se obtienen para los marcos esbeltos (AF, PF y CC) asociados a los vidriados claros (\#1, \#4 y \#6), y dentro de los valores más bajos destacan los casos que incluyen el vidriado \#9, así como el marco de PVC de tipología de abrir (por tener el máximo ancho y un $\mathrm{K}_{\mathrm{m}}$ bajo).

Se distinguen diferencias relativas importantes (24 y $30 \%$ ) entre los índices FS y TV de las aberturas con dimensiones $0.6 \times 1.1 \mathrm{~m}$ y $1.5 \times 1.2 \mathrm{~m}$, respectivamente, mientras que estas diferencias se acortan entre las dimensiones $1.5 \times 1.2$ m y 1.8 x 1.5 m (6 y $8 \%$ ). Incluso, la clasificación de las ventanas en función del factor solar se puede modificar de acuerdo a las dimensiones de las mismas. Por ejemplo, el factor solar de la ventana de PVC de abrir con vidriado \#10 (PA10) es de 0.204 cuando las dimensiones son $0.6 \times 1.1 \mathrm{~m}$, y es de 0.510 cuando son de $1.8 \times 1.5 \mathrm{~m}$. En cuanto a la ventana de aluminio sin RPT, tipología paño fijo, asociada al vidriado 7 (AF7), su factor solar no cambia sensiblemente al modificar las dimensiones (0.399 contra 0.436). En consecuencia, para localidades en las que predomina la demanda de refrigeración sobre la de calefacción, donde el factor solar de las ventanas es una herramienta esencial de mitigación del sobrecalentamiento de los espacios interiores, y considerando las dimensiones $0.6 \times 1.1 \mathrm{~m}$, es más eficiente la ventana PA10 que la AF7; mientras que para las dimensiones $1.8 \times 1.5 \mathrm{~m}$, es más eficiente la ventana AF7 que la PA10. 

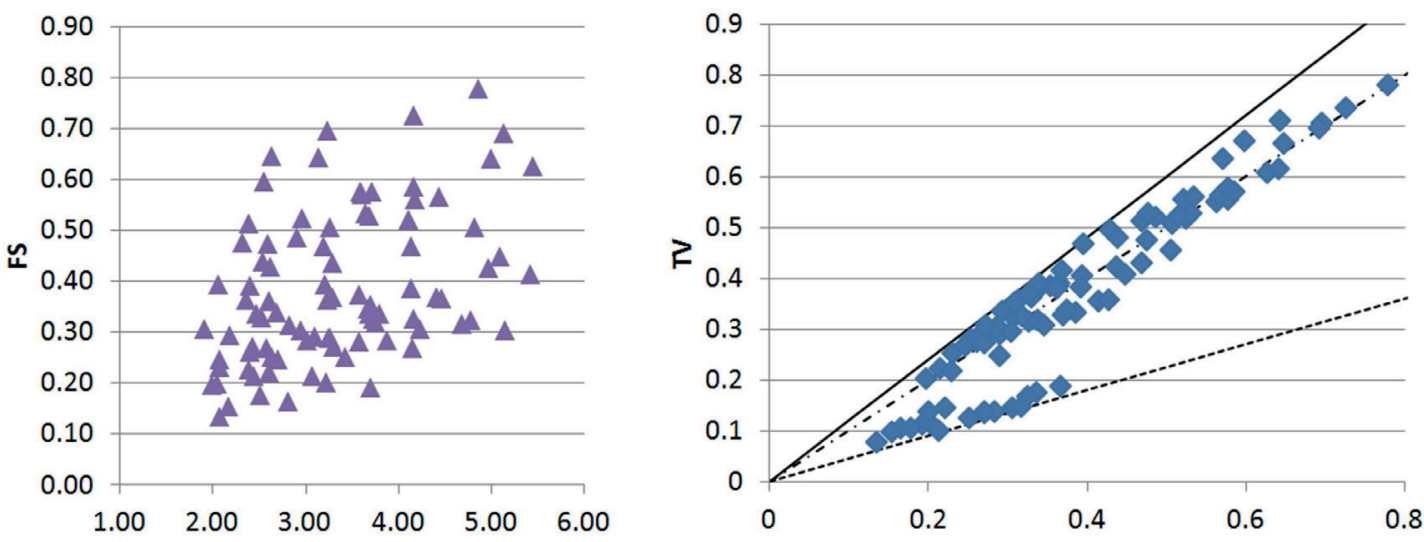

Figura 6. Dispersión entre los índices K y FS (gráfico izquierdo) y dispersión entre los índices FS y TV (gráfico derecho). Fuente: Elaboración de las autoras.

La Figura 5 representa las variaciones del FS de acuerdo a la absortancia solar del marco (para ventanas de dimensiones $1.5 \times 1.2 \mathrm{~m}$ ). Se observan diferencias relativas significativas para los marcos de aluminio corredizo y de abrir (24\%), y para los marcos de aluminio con RPT y PVC de abrir (18 y 21 $\%)$. Esto se explica por la combinación entre una elevada conductancia (sobre todo para los primeros) y la fracción importante que representa la superficie relativa del marco (entre 26 y $43 \%$ ).

La Figura 6 muestra los gráficos de dispersión entre los índices $\mathrm{K}$ y FS de las ventanas estudiadas, para las dimensiones $1.5 \times 1.2 \mathrm{~m}$. Indica que existe una buena diversidad de combinaciones (K, FS) que permiten elegir ventanas con diferentes características según el clima y la orientación de la fachada. Sin embargo, se advierte que las ventanas difícilmente combinan un factor $\mathrm{K}$ bajo con un factor solar elevado (el caso óptimo para lograr estas características es la ventana PF6, de PVC tipología paño fijo con DVH claro, que obtiene un $\mathrm{K}$ de $2.63 \mathrm{~W} / \mathrm{m}^{2} \mathrm{~K}$ y un FS de 0.65).

En cuanto a la selectividad de las ventanas, que se refiere a la relación entre la transmitancia visible y el factor solar, esta se encuentra entre 0.45 y 1.2: los valores mínimos corresponden a las ventanas con simple vidrio de control solar (\#3) y los valores máximos, a ventanas con vidriado doble de control solar (\#7), especialmente a la tipología paño fijo, por su ancho reducido.

\section{CONCLUSIONES}

Los valores de transmitancia térmica de las ventanas disponibles en el mercado argentino tienen una amplia distribución (desde 6.00 hasta $1.88 \mathrm{~W} / \mathrm{m}^{2} \mathrm{~K}$ ). La transmitancia térmica de la ventana puede ser reducida en hasta un $70 \%$ con respecto a la ventana más comúnmente utilizada. No obstante, incluso las ventanas más aislantes tienen factores K elevados en comparación con las tecnologías existentes en otros países, que alcanzan valores de transmitancia térmica inferiores a $0.8 \mathrm{~W} / \mathrm{m}^{2} \mathrm{~K}$ (Jelle et al., 2012).

En cuanto a los valores de factor solar, se obtiene una escala amplia (desde 0.08 hasta 0.80), con una buena dispersión en relación a los valores de transmitancia térmica, lo cual permite seleccionar la ventana adecuada según la estrategia de ahorro energético en función del clima y de la orientación.

Es interesante notar que el color del marco (asociado a un determinado valor de absortancia solar) puede incidir en hasta un $24 \%$ en el valor del FS total de la ventana, lo cual indica que el marco no solo influye en el FS por la reducción de la superficie transparente, sino que también por su factor solar propio, especialmente para los marcos de alta conductancia.

Los índices de transmitancia visible obtenidos evidencian cierto grado de correlación con el factor solar (valores de selectividad comprendidos entre 0.45 y 1.2); de ahí que limitar el factor solar de las ventanas (para evitar el sobrecalentamiento en climas cálidos) implique limitar también el aprovechamiento de la luz natural para iluminación.

Las variaciones de los índices K, FS y TV, de acuerdo con las dimensiones de la ventana, pueden ser significativas. Por este motivo, un sistema de certificación de eficiencia energética de ventanas no debería utilizar dimensiones estándares. Destaca aquí la importancia del marco en las ventanas de dimensiones reducidas (en nuestro ejemplo de $0.66 \mathrm{~m}^{2}$ ), que ocupa entre 22 y $68 \%$ de la superficie total de la ventana según el ancho que tiene, por lo que su elección influye significativamente los valores de los índices energéticos de la ventana. En cambio, para las 
ventanas con una elevada superficie vidriada (en nuestro ejemplo de $2.7 \mathrm{~m}^{2}$ ), el enfoque deberá estar puesto prioritariamente en la tecnología del vidriado. Para las ventanas de dimensiones medianas (en nuestro ejemplo $1.8 \mathrm{~m}^{2}$ ), ambos componentes deberán ser elegidos cuidadosamente.

Las estrategias para mejorar la eficiencia energética del hábitat construido en relación a las tecnologías de ventana dependen del clima y de la orientación de la fachada. En función de estos parámetros se valorarán valores bajos o altos de los índices energéticos de las ventanas. Los resultados de esta investigación constituyen un aporte al hábitat sustentable, ya que presentan un panorama de las propiedades energéticas de las tecnologías de ventanas disponibles en Argentina, a partir del cual es posible elegir las tecnologías más eficientes desde el punto de vista energético, de acuerdo al contexto edilicio y geográfico analizado. En futuros desarrollos de este trabajo se buscará determinar cuáles son los índices energéticos de ventana ideales para diferentes configuraciones edilicias y zonas climáticas.

\section{AGRADECIMIENTOS}

Consejo Nacional de Investigaciones Científicas y Técnicas - Agencia Nacional de Promoción Científica y Tecnológica, Argentina - Fondo para la Investigación Científica y Tecnológica. Proyectos PICT 2016-1487. Pres. BID y PIP 0728 - CONICET.

\section{REFERENCIAS BIBLIOGRÁFICAS}

ALAM, Jahangir y ISLAM, Mohamed A. Effect of external shading and window glazing on energy consumption of buildings in Bangladesh. Advances in Building Energy Research, 2016, vol. $11, n^{\circ} 2$, pp. 1-13.

ALWETAISHI, Mamdooh. Impact of glazing to wall ratio in various climatic regions: A case study. Journal of King Saud UniversityEngineering Sciences, 2017, vol. 31, n 1, pp. 6-18.

APPELFELD, David; HANSEN, Christian S. y SVENDSEN, Svend. Development of a slim window frame made of glass fibre reinforced polyester. Energy and Buildings, 2010, vol. 42, $n^{\circ} 10$, pp. 1918-1925.

BURATTI, Cinzia; MORETTI, Elisa y ZINZI, Michele. High energyefficient windows with silica aerogel for building refurbishment: experimental characterization and preliminary simulations in different climate conditions. Buildings, 2017, vol. 7, nº 1, p.8.

CARDINALE, Nicola; ROSPI, Gianluca y CARDINALE, Tiziana. Numerical and experimental thermal analysis for the improvement of various types of windows frames and rolling-shutter boxes. International Journal of Energy and Environmental Engineering, 2015, vol. 6, n² 2, pp. 101-110.
DE GASTINES, Maureen; CORREA, Érica y PATTINI, Andrea. Window frame thermal performance simulation. Suitable methods according to climate and technology. Journal of Construction, 2018, vol. 17, $\mathrm{n}^{\circ} 1$, pp. 112-122.

DE GASTINES, Maureen y PATTINI, Andrea. Cálculo de precisión del factor solar de ventanas. En: XL Reunión de Trabajo de la Asociación Argentina de Energías Renovables y Medio Ambiente, 2017, vol. 5, pp. 08.167-08.175.

DE GASTINES, Maureen; VILLALBA, Ayelén y PATTINI, Andrea. Improved model for the thermal performance calculation of nonplanar window frames for building simulation programs. Journal of Building Performance Simulation, 2016, vol. 9, n 6, pp. 633647.

GRANZOTTO, Nicola; BETTARELLO, Federica; FERLUGA, Alessio; MARSICH, Lucia; SCHMID, Chiara; FAUSTI, Patrizio y CANIATO, Marco. Energy and acoustic performances of windows and their correlation. Energy and Buildings, 2017, vol. 136, pp. 189-198

INSTITUTO ARGENTINO DE NORMALIZACIÓN Y CERTIFICACIÓN. IRAM 11603: Acondicionamiento térmico de edificios. 2012

INTERNATIONAL ENERGY AGENCY. Technology Roadmap Energy efficient building envelopes. International Energy Agency, 2013.

JELLE, Bjørn Petter; HYND, Andrew; GUSTAVSEN, Arild; ARASTEH, Dariush; GOUDEY, Howdy y HART, Robert. Fenestration of today and tomorrow: A state-of-the-art review and future research opportunities. Solar Energy Materials and Solar Cells, vol. 96, pp. $1-28,2012$.

LECHOWSKA, Agnieszka A.; SCHNOTALE, JacekA. y BALDINELLI, Giorgio. Window frame thermal transmittance improvements without frame geometry variations: an experimentally validated cfd analysis. Energy and Buildings, 2017, vol. 145, p. 188-199.

MEDINA AGREDA, Iván. Estudios de Transmisión de Calor en Marcos de Ventanas de Edificios Diseñados en México. Tesis doctoral, Instituto Tecnológico de Ciudad Madero, 2009.

RAWAL, Rajan; DIDWANIA, Srijan; SHUKLA, Yash; MANU, Sanyogita y PANCHAL, Purvi. Deployment of energy simulation for design of voluntary window labeling program in india. En: Proceeding of BS2013: 13th Conference of International Building Performance Simulation Association, 2013.

SHARDA, Anuranjan y KUMAR, Sudhir. Statistical evaluation of u-value of a window with inter-pane blinds. International Journal of Ambient Energy, 2016, vol. 37, n² 4, pp. 384-397.

THALFELDT, Martin; KURNITSKI, Jarek y VOLL, Hendrik. Detailed and simplified window model and opening effects on optimal window size and heating need. Energy and Buildings, 2016, vol. 127, pp. 242-251.

TRZASKI, Adrian y RUCINSKA, Joanna. Energy labeling of windows-possibilities and limitations. Solar Energy, 2015, vol. 120, pp. 158-174. 
VAN DEN BOSSCHE, Nathan, BUFFEL, Lisa y JANSSENS, Arnold. Thermal optimization of window frames. Energy Procedia, 2015, vol. 78 , pp. $2500-2505$.

VENHAUS-HELD, Manuel; ALÍAS, Herminia María y JACOBO, Guillermo José. Las envolventes constructivas en la construcción no convencional de edificios del nordeste argentino y el problema de los puentes térmicos. Hábitat Sustentable, 2017, vol. 7, n 1, pp. 24-31.

WRIGHT, John L. y MCGOWAN, Alexander. Calculating the solar heat gain of window frames. ASHRAE Transactions, 1999, vol. $105, n^{\circ} 2$, pp. 1011-21.

YAZDANIAN, Mehrangiz y KLEMS, Joseph. Measurement of the exterior convective film coefficient for windows in low-rise buildings. ASHRAE Transactions, 1994, vol. 100, Part 1.

ZAJAS, Jan y HEISELBERG, Per. Parametric study and multi objective optimization of window frame geometry. Building Simulation, 2014, vol. 7, n 6, pp. 579-593. 